\title{
Erratum to: A genome-wide scan for quantitative trait loci affecting serum glucose and lipids in a White Duroc $\times$ Erhualian intercross $\mathbf{F}_{2}$ population
}

\author{
Rongrong Chen $\cdot$ Jun Ren $\cdot$ Wanbo Li $\cdot$ Xiang Huang $\cdot$ \\ Xueming Yan • Bin Yang $\cdot$ Yinggong Zhao • \\ Yuanmei Guo $\cdot$ Huirong Mao $\cdot$ Lusheng Huang
}

Published online: 20 March 2010

(C) Springer Science+Business Media, LLC 2010

\section{Erratum to: Mamm Genome (2009) 20(6):386-392 \\ DOI 10.1007/s00335-009-9190-9}

In the above-mentioned article, published in this issue of Mammalian Genome, seven errata should be made. They are:

1. In the first sentence of the 'Phenotype recording' section on p. 387, the correct description should be ' $\mathbf{4 1 2}$ barrows and 348 gilts'.

2. In the second sentence on p. 390 , the correct text to mention the QTL on SSC2 should read: The White Duroc alleles at these loci were associated with increased phenotypic values of TC and LDL-C.

3. In the second paragraph on p. 390, the right statement of the QTL for TG and HDL-C on SSC5 should read: At these two QTL the alleles that decrease phenotypic values were inherited from the Erhualian breed.

4. In the last paragraph but one on p. 390, the correct presentation for the QTL for HDL-C and TG on SSC9 should read: At the two QTL, the QTL alleles that increase phenotypic values were inherited from the White Duroc breed.

5. In the first sentence on p. 391, the right description of the QTL for HDL-C and TC on SSC11 should be 'the White Duroc alleles that increase phenotypic values'.

6. In the 'QTL for glucose and GSP' section on p. 391, the second sentence should be revised as: The alleles that increase phenotypic values came from the White Duroc breed.

7. In Table 1 on p. 388, the superfluous words of 'QTL Express' in the third line should be deleted.

The online version of the original article can be found under doi:10.1007/s00335-009-9190-9.

R. Chen · J. Ren · W. Li $\cdot$ X. Huang $\cdot$ X. Yan $\cdot$ B. Yang ·

Y. Zhao $\cdot$ Y. Guo $\cdot$ H. Mao $\cdot$ L. Huang $(\bowtie)$

Key Laboratory for Animal Biotechnology of Jiangxi Province

and the Ministry of Agriculture of China, Jiangxi Agricultural

University, Nanchang 330045, China

e-mail: Lushenghuang@hotmail.com 